Communications in Physics, Vol. 31, No. 3 (2021), pp. 301-312

DOI:10.15625/0868-3166/15885

\title{
GAS SENSING PROPERTIES OF NANOJUNCTION OF NETWORKED ZnO NANOWIRES UNDER THE CORRELATION BETWEEN OPERATING TEMPERATURE AND UV RADIATION
}

\author{
NGUYEN MANH HUNG ${ }^{1}$, CHU MANH HUNG $^{2}$ AND NGUYEN VAN HIEU ${ }^{3, \dagger}$ \\ ${ }^{1}$ Department of Materials Science and Engineering, \\ Le Quy Don Technical University, Hanoi, Vietnam \\ ${ }^{2}$ International Training Institute for Materials Science, \\ Hanoi University of Science and Technology, Hanoi, Vietnam \\ ${ }^{3}$ Faculty of Electrical and Electronic Engineering, Phenikaa University, Hanoi, Vietnam \\ E-mail: †hieu.nguyenvan@phenikaa-uni.edu.vn
}

Received 22 February 2021

Accepted for publication 24 March 2021

Published 22 April 2021

\begin{abstract}
The networked $\mathrm{ZnO}$ nanowires (NWs) are synthesized by thermal evaporation at $900{ }^{\circ} \mathrm{C}$, using a mixture of $\mathrm{ZnO}$ and graphite. The morphology, crystalline structure, and chemical composition of the NWs are evaluated by field-emission scanning electron microscopy, $X$-ray diffraction, and energy-dispersive spectrum. The $\mathrm{NO}_{2}$ gas-sensing properties of a networked $\mathrm{ZnO} \mathrm{NWs-based}$ sensor are considered in a correlation between the operating temperature and $U V$ radiation with various operating temperatures as well as UV intensities. The results reveal that the sensing properties of the UV-illuminated sensor measured at room temperature are comparable to those of the heated sensor. The results also indicate that the UV intensity affects strongly both the response and the sensing kinetic of the sensor at all operating temperatures. Furthermore, based on a systematic investigation of the sensing performance of the sensor under both UV illumination and thermal activation, a model to explain the role of UV illumination is also proposed.
\end{abstract}

Keywords: UV illumination; $\mathrm{ZnO}$ nanowires sensor; $\mathrm{NO}_{2}$ sensing; UV-illuminated sensor. Classification numbers: 07.07.Df; 81.07.Gf. 


\section{INTRODUCTION}

Nowadays, the rapid development of various fields, including artificial intelligence, the Internet of Things (IoT), and nanomaterials have resulted in the need to develop portable multifunctional devices. Besides, since the concerns of air pollution have increased worldwide, the hazardous gas-detecting function should be integrated into such devices. From the literature, there are many kinds of gas sensors with various gas-sensing principles [1-3]. Among them, chemoresistive gas sensor shows an outstanding performance owing to its lightweight, small size, and simple structure. The gas-detecting mechanism of a chemoresistive gas sensor is based on the resistance change of the gas sensing-material layer under the exposure of target gas. Therefore, the adsorption/desorption processes of the target gas and oxygen molecules in the environment onto the surface of the sensing layer play an important role in controlling the sensing performance. Unfortunately, the sensing layer is usually made of metal oxide semiconductors (MOSs), which need to be thermally activated to adsorption/desorption reactions take place effectively. Generally, the operating temperature of MOS sensors is in the range of $150-400^{\circ} \mathrm{C}$ [4-6]. Because of such high operating temperatures, the properties of the MOS sensors are unstable due to the particle agglomeration phenomenon. Moreover, high operating temperature leads to a series of unfavorable issues, such as high-power consumption, incompatibility of flexible substrates, or a high risk of flammable and/or explosive environments. Therefore, a high operating temperature is a key obstacle that hinders MOS sensors to be integrated into portable electronic devices. In order to address this issue, various solutions have been proposed to reduce the operating temperature, such as morphological/structural modification of nanomaterials [7,8], ultra-violet (UV) and/or visible light illumination [9,10], doping [11], self-heating [12], etc. While the repeatability of the fabrication process is a significant limitation of almost all the above solutions, UV illumination provides a stable technological solution to obtain a low-temperature gas sensor. In recent years, many reports have indicated that thermal activation could be replaced by UV illumination effectively. The UV-illuminated sensors show outstanding sensing performance toward both reducing gases and oxidizing gases at low-temperature, even room temperature (RT). Interestingly, although some target gases $\left(\mathrm{CH}_{4}, \mathrm{CO}\right)$ are known to be difficult to detect, even at high temperatures, some reports claim that these gases could be detected at low temperatures under UV illumination [13, 14]. Moreover, B. P. J. de Lacy Costello et al. reported that the control of the UV intensity is a simple and convenient way to achieve selectivity. In addition to the optimization of the sensing properties under UV illumination as discussed above, various mechanisms are also proposed to explain the unique and interesting performances of the UV-illuminated sensors. The most commonly provided explanations are: (i) the formation of photo-generated electrons that make the adsorption/desorption processes take place more favorably [15]; (ii) the dissociation of target gases under UV radiation into the components that is favorable to adsorb [14]; (iii) the photon-gas interaction, which promotes the desorption of gas molecules from the surface of the sensing layer [16,17]; (iv) and the modulation of the depletion layer width that aims to obtain nanosize effect [15]. Although all the above explanations are related to various aspects of the sensing mechanism, a complete and deeper understanding of the sensing mechanism under UV

illumination remains unclear. Therefore, theoretical and experimental investigations need to be further conducted to give reliable and precise assessments. 
In this study, we report the sensing behavior of networked $\mathrm{ZnO} \mathrm{NWs}$ toward $\mathrm{NO}_{2}$ gas under the correlation between the operating temperature and UV radiation. The results illustrate that the UV-illuminated sensor could operate effectively at room temperature without heating. Besides, based on a systematic investigation of the sensing performance of the networked $\mathrm{ZnO}$ NW sensor under both UV radiation and thermal activation, a model is proposed to explain the role of UV radiation in control of the sensing behavior of the sensor under UV illumination at various temperatures. Therefore, the aim of this study is not to maximize the response of the sensor that will be considered in future works.

\section{EXPERIMENT}

\section{II.1. Sensor fabrication}

An oxidized-silicon substrate integrated with a comb-type platinum electrode (silicon substrate) was used to fabricate the networked $\mathrm{ZnO} \mathrm{NW}$ sensor. The fabrication procedure was schematically illustrated in Fig. 1. First, a cleaned-silicon substrate was sputtered with a $5 \mathrm{~nm}$ gold layer as a catalyst. Then $0.3 \mathrm{~g}$ powder consisting of $\mathrm{ZnO}$ and $\mathrm{C}$ (ratio 1:1) was placed into a quartz tube furnace with the prepared substrate. The distance between the powder and the substrate was controlled at $4 \mathrm{~cm}$. The growth procedure was started by making a vacuum value of $1.8 \times 10^{-1}$ Torr with the presence of a $30 \mathrm{sccm}$ argon gas flow in the quartz tube using a rotary pump. When the vacuum achieved the desired value, the furnace was heated to reach $900^{\circ} \mathrm{C}$ with a heating rate of $30^{\circ} \mathrm{C} / \mathrm{min}$. During the heating stage, $1 \mathrm{sccm}$ oxygen was supplied to the quartz tube when the furnace temperature obtained $820^{\circ} \mathrm{C}$. After achieving the necessary temperature, the furnace was remained at that temperature within $60 \mathrm{~min}$ for the growth of $\mathrm{ZnO}$ nanowires. During the cooling process, the oxygen flow was shut when the furnace cooled down to $700^{\circ} \mathrm{C}$. Finally, when the temperature of the furnace reached room temperature, the sensor was removed from the quartz tube and stored for further analyses.
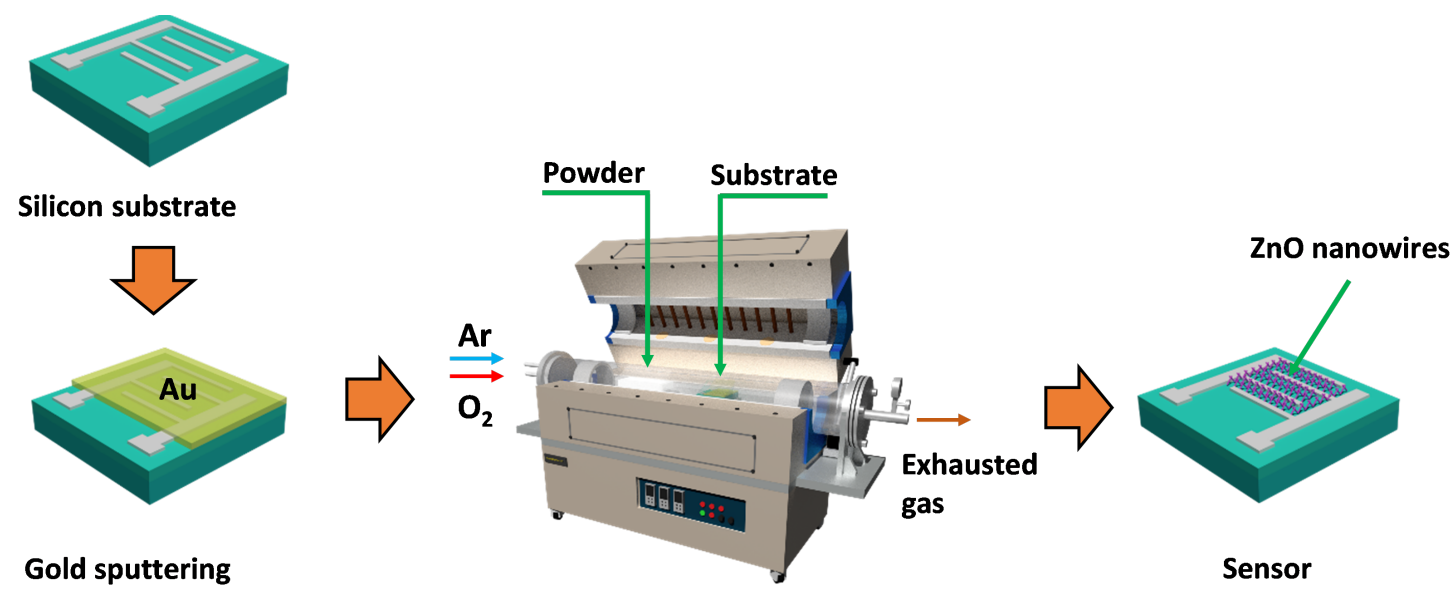

Fig. 1. Schematic illustration of the fabrication process of the networked $\mathrm{ZnO}$ nanowires sensor. 


\section{II.2. Material characterizations and gas-sensing property measurement}

The morphology of the $\mathrm{ZnO}$ NWs was observed by a field-emission scanning electron microscopy (FE-SEM, JSM-7600F), while the chemical composition was analyzed by energy dispersive spectroscopy (EDS) equipped with SEM. The crystalline structure of nanowires was examined by X-ray diffraction (XRD, Bruker D5005) using $\mathrm{Cu} \mathrm{K} \alpha$ radiation $(\lambda=0.154056 \mathrm{~nm})$ with a scanning rate of $0.015 \%$.

The gas-sensing properties of the sensor were investigated with a home-made measurement system. $\mathrm{NO}_{2}$ and dry air flows were controlled accurately by mass flow controllers (MFCs) and were mixed to create desired $\mathrm{NO}_{2}$ concentrations. To ensure the stability of the gas-sensing test, the total flow rate of the gas mixture was maintained at $300 \mathrm{sccm}$. The response of the sensor $(S)$ was determined by $S=R_{g} / R_{a}$, where $R_{g}$ and $R_{a}$ are the resistance values of the sensor in the gas mixture and dry air, respectively. The response time was estimated technically by the time to reach $\sim 90 \%$ maximal response level, whereas the recovery time was for $\sim 90 \%$ the value of $R_{a}$ (or total time of recovery process if the corresponding recovery process is incomplete).

To consider the effect of UV radiation, the sensor was illuminated from a UV lamp (BLE220B, Spectroline, USA) at a fixed distance. The UV intensity can be changed using shutters of various diameters. The real values of different UV intensities on the surface of the sample were measured by a commercial UV detector (XRP-3000, Spectroline, USA).

\section{RESULTS AND DISCUSSION}

\section{III.1. Structural and morphological characteristics}

Figure 2(a) shows that $\mathrm{ZnO}$ NWs grow densely on the electrode regions. In the gap region between two electrodes, the NWs connect to form networked NWs with a lower density. The SEM image with a higher magnification displays a narrow distribution of the diameters of the synthesized NWs with a characteristic diameter of $\sim 35 \mathrm{~nm}$ (Fig. 2(b) and the inset). The mechanism of vapor-liquid-solid growth of $\mathrm{ZnO} \mathrm{NWs}$ is also confirmed with the presence of the catalytic gold particle at the top of the nanowire (the inset). The 1D morphologies of NWs lead to preferential orientations of (100) and (101) lattice planes that are confirmed by X-ray diffraction in Fig. 2(c). From the literature, isotropic $\mathrm{ZnO}$ particles are characteristic with comparable diffraction intensities of (100), (002), and (101) peaks [18,19]. In this study, ZnO NWs display the wurtzite crystalline structure (JCPDS 36-1451) with narrow and sharp diffraction peaks, which reveals a high crystalline quality. There are no peaks of contaminants that could be detected under the resolution of the XRD equipment. This result is in good agreement with that of the EDS spectrum (Fig. 2(d). Furthermore, atomic percentages of $\mathrm{Zn}$ and $\mathrm{O}$ elements estimated from EDS show a non-stoichiometry composition of $\mathrm{ZnO}$ with an $\mathrm{O}$ deficiency that creates the n-type semiconducting property of this metal oxide. Generally, the various investigations prove that a networked $\mathrm{ZnO}$ NWs-based sensor was fabricated successfully.

\section{III.2. Gas sensing properties and sensing mechanism}

The dynamic responses of the networked $\mathrm{ZnO}$ NWs-based sensor upon exposure of $5 \mathrm{ppm}$ $\mathrm{NO}_{2}$ are investigated both in dark and under UV irradiation with different intensities at $30^{\circ} \mathrm{C}$ (Fig. 3(a)). For comparison, the responses of the sensor toward $5 \mathrm{ppm} \mathrm{NO}_{2}$ at various operating temperatures are also examined. The key results are summarized as follows: 


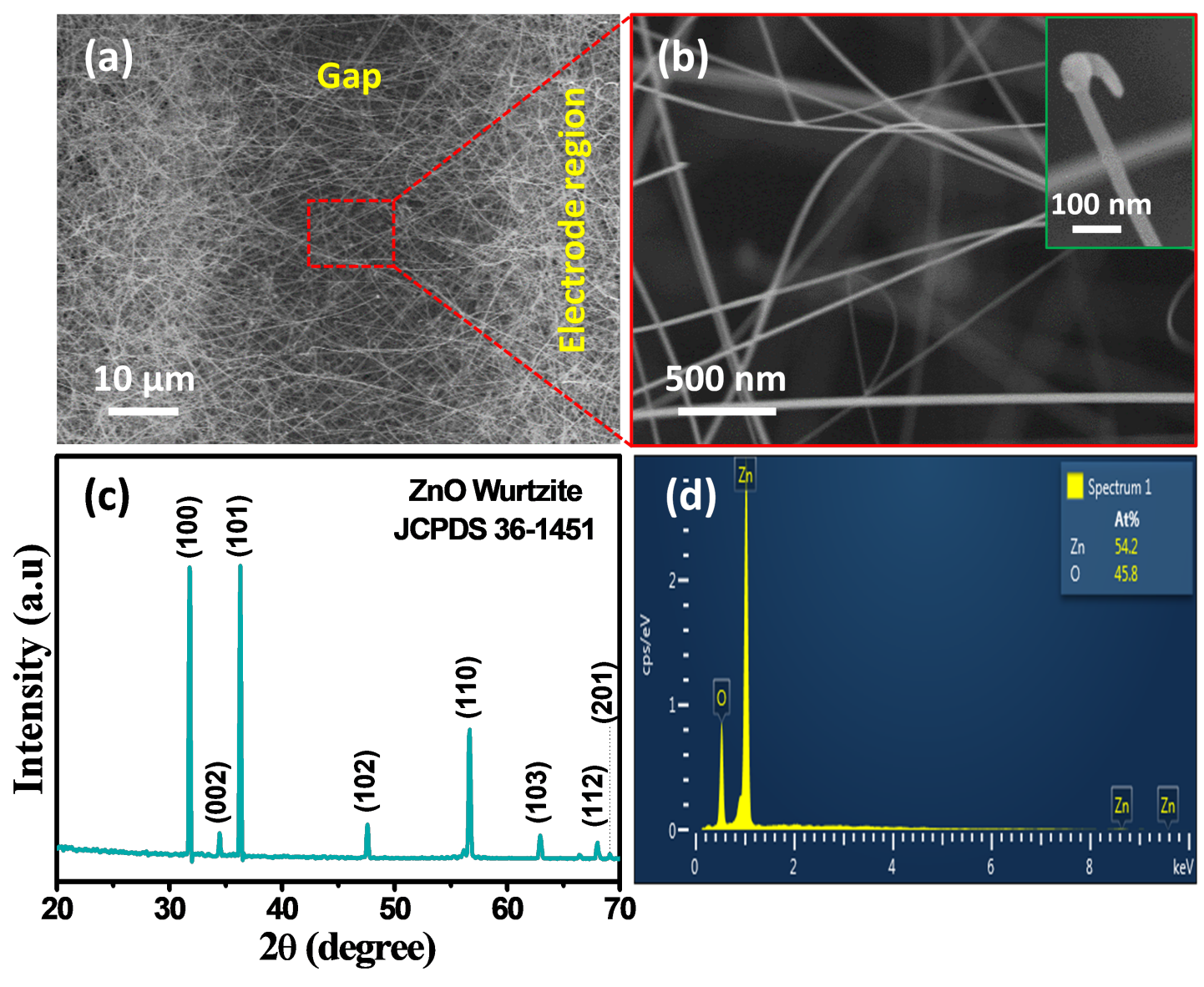

Fig. 2. (a, b) SEM images with different magnifications of networked $\mathrm{ZnO}$ nanowires grown by thermal evaporation technique. The inset shows a typical nanowire with a gold particle on the top. (c) X-ray diffraction and (d) EDS spectra of $\mathrm{ZnO}$ nanowires.

i) The sensor showed the extremely slow response and recovery kinetics with a low response in dark at $30^{\circ} \mathrm{C}$. On the contrary, the response and response/recovery kinetics are enhanced significantly in the condition of either UV irradiation or heating.

ii) The responses of the sensor under various UV intensities (excluding the response at the UV intensity of $1350 \mu \mathrm{W} / \mathrm{cm}^{2}$ ) are larger than those of the sensor supported by heating below $200^{\circ} \mathrm{C}$ (Fig. 3(c)). Besides, there is an optimal value of UV intensity to achieve the maximal response of the UV-illuminated sensor.

iii) The response/recovery times of the sensor under different UV intensities are shortened proportionally along with UV intensities. Interestingly, when the sensor was illuminated with an intensity of $1350 \mu \mathrm{W} / \mathrm{cm}^{2}$, the recovery time of the sensor is shorter than that of the sensor measured at all of the investigated temperatures (Fig. 3 (a, b, d)).

It should be noted that the response $(S)$ of the sensor was determined by $S=R_{g} / R_{a}=$ $\left(R_{a}+\Delta R\right) / R_{a}$. Therefore, $\Delta R=R_{g}-R_{a}$ shows the supply level of electrons to the surface for 

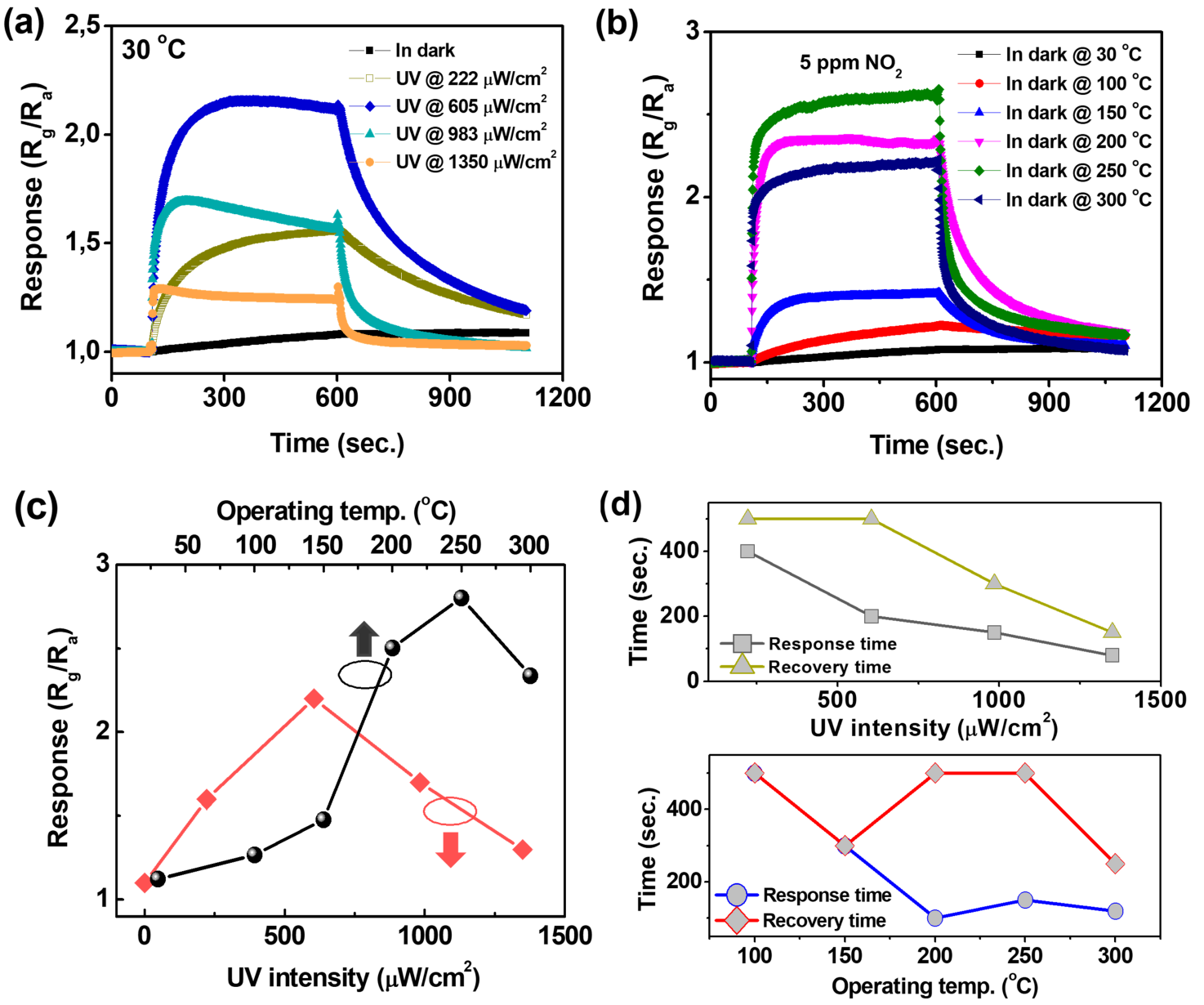

Fig. 3. The transient response curves of the networked $\mathrm{ZnO}$ nanowires-based sensor toward $5 \mathrm{ppm} \mathrm{NO}_{2}$ : (a) in dark and under various UV intensities at $30^{\circ} \mathrm{C}$ and (b) at different operating temperatures in dark. Summaries of the (c) responses and (d) response/recovery times derived from (a) and (b).

the adsorption process. While the response of the sensor depends on both the base resistance $\left(R_{a}\right)$ and the surface adsorption $(\Delta R)$, the response/recovery kinetics depend on only the adsorption/desorption processes of the gases on the surface of the sensing layer. The thermal energy is small when the sensor is measured in dark and at $30^{\circ} \mathrm{C}$. Therefore, surface reactions (1), (2), and (3) related to the adsorption/desorption of oxygen gas $\left(\mathrm{O}_{2}\right)$ /oxygen ion $\left(\mathrm{O}_{2}^{-}\right)$and $\mathrm{NO}_{2}$ molecules are difficult to take place:

$$
\begin{aligned}
\mathrm{O}_{2}+e^{-} & \longrightarrow \mathrm{O}_{2}^{-} \\
\mathrm{NO}_{2(\text { gas })}+e^{-} & \longrightarrow \mathrm{NO}_{2}^{-} \\
\mathrm{NO}_{2 \text { (gas) }}+\mathrm{O}_{2}^{-} & \longrightarrow \mathrm{NO}_{2}^{-}+\mathrm{O}_{2 \text { (gas) }}
\end{aligned}
$$


As a result, the sensor reveals extremely slow response/recovery kinetics (Fig. 3 (a, d)). Under either UV illumination or heating, the sensor showed significantly faster response/recovery kinetics compared to the sensor in dark at $30^{\circ} \mathrm{C}$ (Fig. 3(a,b)). These observations indicate that UV illumination can promote the adsorption/desorption of the gases, which is usually supported by heating. The higher the intensity is, the faster the response and recovery kinetics are. To further understand the enhanced sensing mechanism of the sensor under UV radiation, we examined the effect of UV illumination on the responses of the networked $\mathrm{ZnO}$ nanowiresbased sensor measured at various operating temperatures (Fig. 4). As one can see that the presence of UV illumination shortens the response/recovery times of the sensor in all of the examined temperatures. However, the effects of UV irradiation on the responses and sensing kinetics decrease gradually along with the increase of the operating temperature. While the differences in the response curves are clear at below $250^{\circ} \mathrm{C}$ (Fig. 3(a) and Fig. 4 (a,b)), the response curves of the sensor under various UV intensities at $250^{\circ} \mathrm{C}$ showed an insignificant difference (Fig. 4(c)). These results indicate that the UV illumination is effective at low temperatures (below $250^{\circ} \mathrm{C}$ ), while it is little sense at higher operating temperatures. Similar observations were recorded when the sensor was examined at a fixed intensity of 1350 $\mu \mathrm{W} / \mathrm{cm}^{2}$ with various operating temperatures (Fig. 5). At low operating temperatures $\left(30^{\circ} \mathrm{C}\right.$ and $\left.100^{\circ} \mathrm{C}\right)$, the sensor displays the fast response/recovery kinetics. However, response/recovery kinetics became significantly slower when the operating temperature increases further. Besides, the response of the sensor is enhanced clearly at high operating temperatures $\left(\geq 150^{\circ} \mathrm{C}\right)$ compared to that of the sensor at low operating temperatures (a)

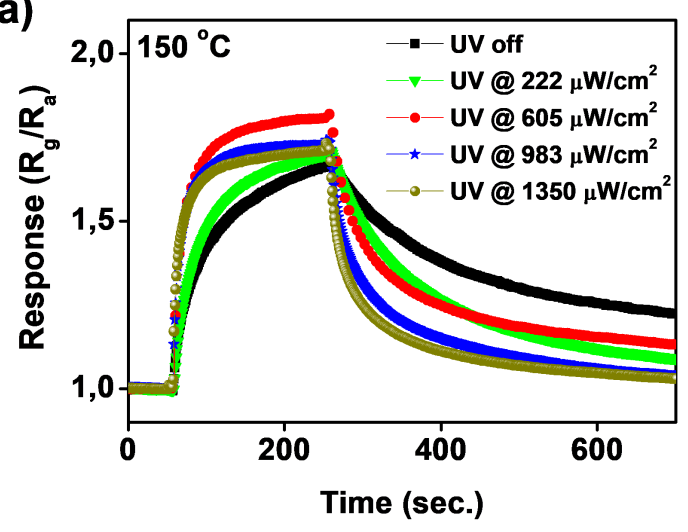

(b)

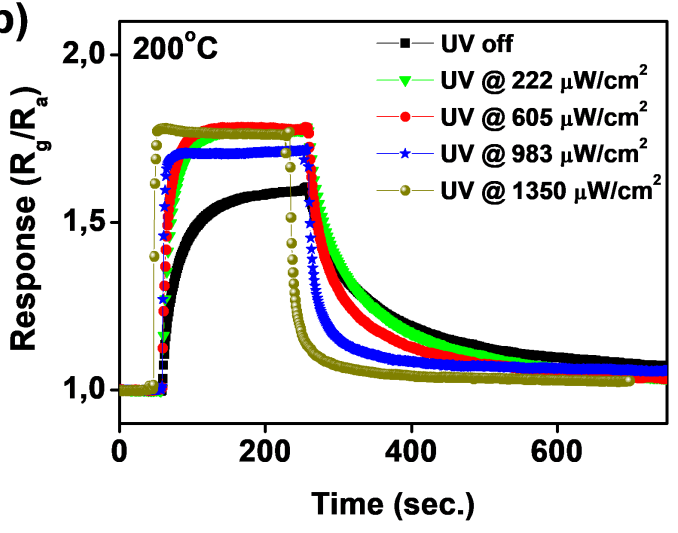

(c)

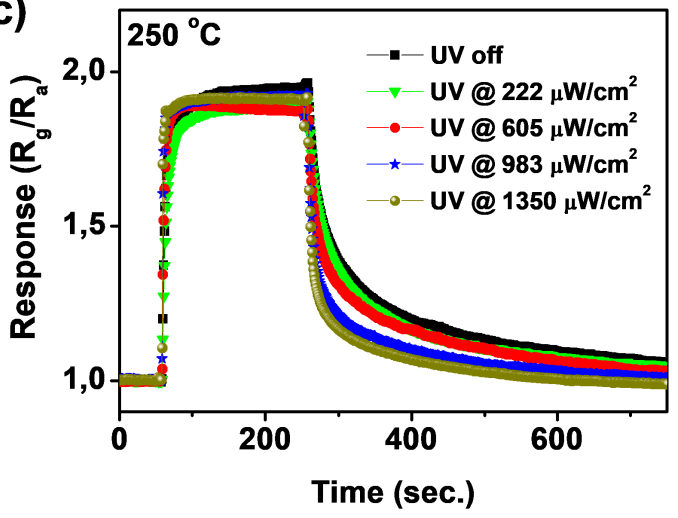

Fig. 4. The effect of UV illumination on the responses of the networked $\mathrm{ZnO}$ nanowires sensor measured at various operating temperatures. 
$\left(<150^{\circ} \mathrm{C}\right)$. These observations further confirm that the effect of UV illumination is dominant at low operating temperatures, while that of heating is overwhelming at high operating temperatures. The existing states of oxygen on the surface of MOS known is to depend on the temperature. Physical adsorption is in low-temperature regions, whereas chemical adsorption at higher temperatures. Therefore, based on the observed results, the UV-dependent recovery kinetic of the sensor at all operating temperatures should be ascribed to the enhancement of gas release by UV photons. The amount of energy that a $\mathrm{NO}_{2}$ molecule receives through the collision with UV photons, along with thermal energy, allows the $\mathrm{NO}_{2}$ desorption to become more effective, thereby the recovery process. It can be observed that the recovery time reduces significantly with an increase of UV intensity at all operating temperatures (Fig. 3(a) and Fig. 4). It is noteworthy that the UV intensity is proportional to the number of UV photons. Therefore, the higher the intensity is, the more much the UV photons collide with $\mathrm{NO}_{2}$ molecules. As a result, a larger amount of $\mathrm{NO}_{2}$ molecules could be desorbed from the surface of the $\mathrm{ZnO}$ nanowires at a higher UV intensity. In this study, the sensor is irradiated by a $365 \mathrm{~nm}$ wavelength, which corresponds to a photon energy of $\sim 3.39 \mathrm{eV}$. In the meanwhile, the theoretical calculations indicate that the maximal binding energies of $\mathrm{O}_{2}$ and $\mathrm{NO}_{2}$ molecules on the (100) preferential plane are $2.08 \mathrm{eV}$ [20] and $0.36 \mathrm{eV}$ [21], respectively. The energy of the UV photon is large enough to desorb $\mathrm{NO}_{2}$ and $\mathrm{O}_{2}$ molecules directly from the surface of $\mathrm{ZnO}$ nanowires, thereby shortening significantly the recovery times of the sensor under UV radiation. At a fixed UV intensity, the slower responses and/or recoveries of the sensor operated at at high operating temperatures compared to those of the sensor at RT in Fig. 5 should be considered by the change of the existing state of oxygen ions on the surface of $\mathrm{ZnO}$ nanowires as discussed above, as well as the changes in the reactions of $\mathrm{NO}_{2}$ with the surface and pre-adsorbed oxygen ions. Generally, more detailed discussions are necessary, but they should be considered in another work. In this study, the recovery time of the sensor was still shortened when the sensor is illuminated by UV radiation at all operating temperatures. Therefore, we safely claim that UV illumination can shorten the recovery time through the direct desorption of $\mathrm{NO}_{2}$ molecule from the surface of $\mathrm{ZnO}$ nanowires. The effects of UV radiation on the response and response kinetics are more complicated and will be discussed below.

The enhanced response and response kinetics of the UV-illuminated sensor are considered under some aspects. Fig. 6 shows the resistance change of the networked $\mathrm{ZnO}$ nanowires sensor against UV radiation. It should be noted that the energy of the UV photon is $\sim 3.39 \mathrm{eV}$, whereas the bandgap of $\mathrm{ZnO}$ is in a $3.1-3.37 \mathrm{eV}$ range [22,23]. Therefore, the resistance reduces rapidly when the UV lamp is turned on. This behavior is related to the band-to-band transitions. However, a slow and small increase in the resistance is also observed as a second stage under UV illumination, which is ascribed to the re-adsorption of a small number of oxygen molecules, which are pre-desorbed by UV photons. Similarly, the recovery process takes time of the order of hours. Therefore, this process should be considered by not only band-to-band transitions, but also the surface processes. The recovery of resistance includes two stages: the first stage with a fast recovery, which is contributed by band-to-band transitions, while the extremely slow second stage is ascribed to the re-adsorption of oxygen molecules. Clearly, a new equilibrium between the adsorption/desorption processes of oxygen ions needs a long time to achieve. A. Giberti, et al. also reported a similar observation regarding the overwhelming role of surface processes in the resistance change of $\mathrm{WO}_{3}$ nanopowder under UV illumination [16]. Therefore, the effect of UV radiation on the response behavior of the sensor is proposed as follows: 


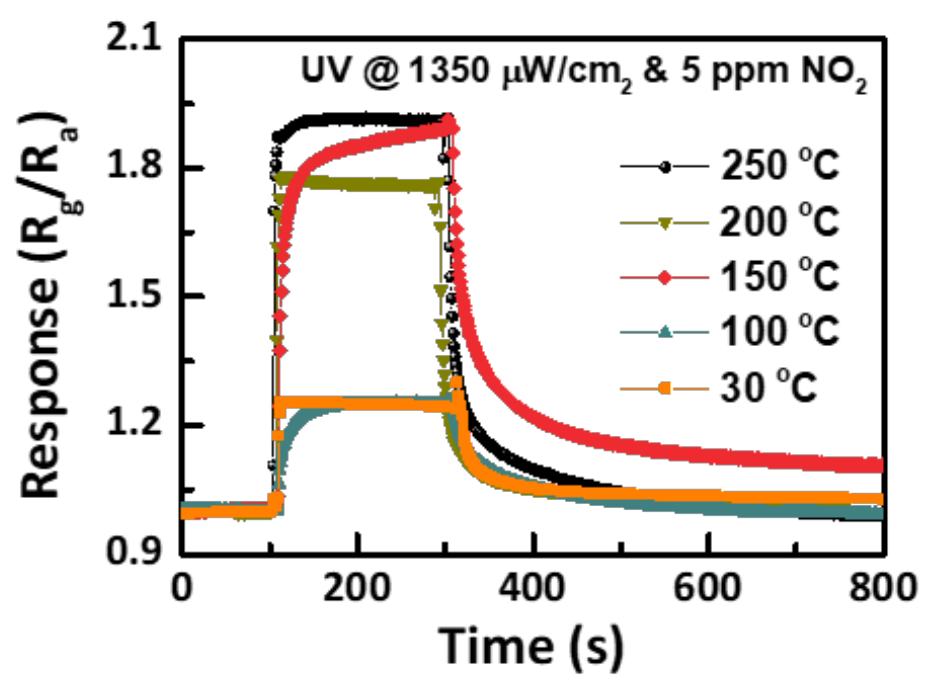

Fig. 5. The effect of various operating temperatures on the sensing behavior of the UV-illuminated sensor at a fixed intensity of $1350 \mu \mathrm{W} / \mathrm{cm}^{2}$.

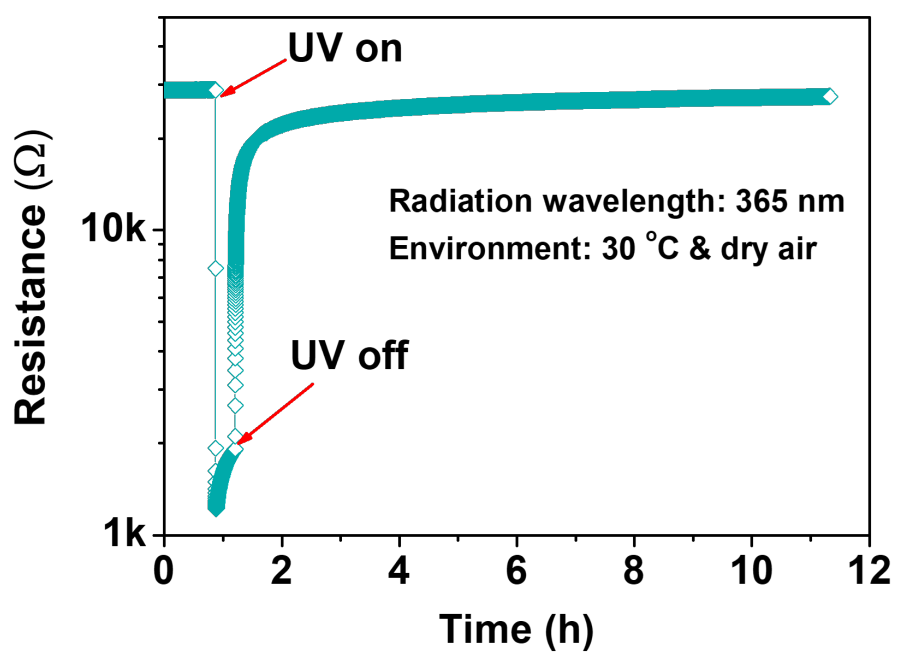

Fig. 6. The resistance change of the networked $\mathrm{ZnO}$ nanowires-based sensor against UV irradiation.

i) The adsorption sites increased significantly due to the desorption of oxygen ions under UV illumination compared to the adsorption site density in dark. The surface reactions under UV radiation are proposed as follows:

$$
\begin{aligned}
h v & \longrightarrow h^{+}+e_{(h v)}^{-} \\
\mathrm{O}_{2}^{-}+h^{+} & \longrightarrow \mathrm{O}_{2(\mathrm{gas})}
\end{aligned}
$$




$$
\begin{aligned}
\mathrm{O}_{2(\text { gas })}+e_{(h v)}^{-} & \longrightarrow \mathrm{O}_{2(h v)}^{-} \\
\mathrm{NO}_{2(\text { gas })}+e_{(h v)}^{-} & \longrightarrow \mathrm{NO}_{2(h v)}^{-} \\
\mathrm{NO}_{2(\text { gas })}+\mathrm{O}_{2(h v)}^{-} & \longrightarrow \mathrm{NO}_{2(h v)}^{-}+\mathrm{O}_{2 \text { (gas) }}
\end{aligned}
$$

As a result, more $\mathrm{NO}_{2}$ molecules were adsorbed onto the surface of $\mathrm{ZnO}$ nanowires, thereby leading to a larger change of energy barrier $\Delta q V b(h v)$ compared to that of energy barrier in dark $\Delta q V b$ (Fig. 7). These surface reactions are responsible for the higher response of the sensor under UV illumination as shown in equation (9) [24]:

$$
S=R_{g} / R_{a} \sim \exp [\Delta q V b / k T],
$$

where $\Delta q V b$ is the change in the potential barrier height at nanojunction between two nanowires, $k$ is Boltzmann, and $T$ is the absolute temperature.

(a)

\section{In dark}

Depletion layer

\section{In air}

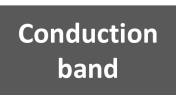

$\ln \mathrm{NO}_{2}$
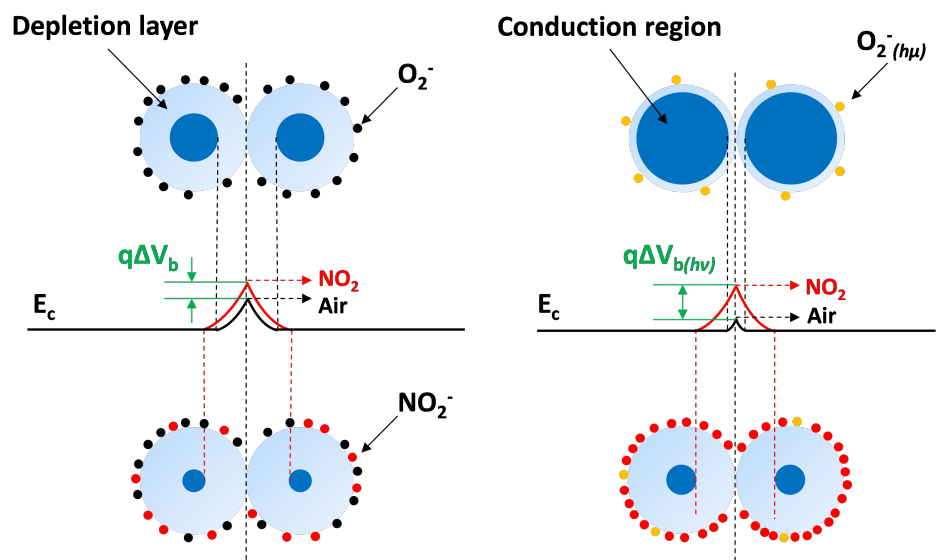

Fig. 7. The illustration of surface reactions and the corresponding changes of energy barriers of $\mathrm{ZnO}$ nanowires under exposures of dry air and $\mathrm{NO}_{2}$ gas at room temperature: (a) in dark and (b) under UV illumination.

ii) The presence of UV-generated electrons leads to an effective supply of UV-generated electrons to the surface reactions, including the adsorption reaction of $\mathrm{NO}_{2}$ molecules. Therefore, along with the increased adsorption sites, the effective supply of UVgenerated electrons allows $\mathrm{NO}_{2}$ molecules to adsorb more easily and effectively, thereby creating a faster and higher response.

iii) The maximal response of the sensor is reached when two following factors are satisfied: (1) a large amount of $\mathrm{NO}_{2}$ molecules are adsorbed onto the surface of the 
nanowires to obtain a large $\Delta R$; (2) the depletion layer width of nanowires is comparable to the size that reaches the nanosize effect. In the meanwhile, both two these factors strongly depend on the UV intensity. Therefore, the sensing response shows a peak at a UV intensity of $605 \mu \mathrm{W} / \mathrm{cm}^{2}$ (Fig. $3(\mathrm{a}, \mathrm{c})$ ). The response decreases if the UV intensity is far away from the optimal UV value is due to the reduced adsorption of $\mathrm{NO}_{2}$ molecules and/or the inefficient modulation of the depletion layer width.

\section{CONCLUSIONS}

The effect of UV radiation on the sensing behavior of the networked $\mathrm{ZnO}$ nanowires-based sensor was investigated. The UV-illuminated sensor has been proven to be a good alternative to the heated sensor. The UV-illuminated sensor could obtain faster response/recovery kinetics with a higher response if the UV intensity is chosen suitably. Besides, a systematical investigation of the sensing behavior of the networked $\mathrm{ZnO}$ nanowires-based sensor under both $\mathrm{UV}$ radiation and heating suggests an enhanced sensing mechanism, which is based on the UV photon-supported desorption of gases; the effective supply of UV-generated electrons to the surface reactions; and the modulation of the depletion layer width. The results of this study are another approach to understand further the sensing mechanism of the MOS sensors under UV radiation, which is expected to pave the way to develop room-temperature gas sensors.

\section{ACKNOWLEDGMENTS}

This work was supported by the Vietnam National Foundation for Science and Development (NAFOSTED) under grant number 103.02-2017.25.

\section{REFERENCES}

[1] Z.P. Khlebarov, A.I. Stoyanova, D.I. Topalova, Sens. Actuators B Chem. 8 (1992) 33.

[2] K. Yadav, S.K. Gahlaut, B.R. Mehta, J.P. Singh, Appl. Phys. Lett. 108 (2016) 071602.

[3] D. K. Aswal, S. K. Gupta, Science and technology of chemiresistor gas sensors, Nova Publishers, 2007.

[4] A. Mirzaei, S.S . Kim, H. W. Kim, J. Hazard. Mater. 357 (2018) 314.

[5] S. Mahajan and S. Jagtap, Appl. Mater. Today 18 (2020) 100483.

[6] Z. Li, Z. Yao, A. A. Haidry, T. Plecenik, L. Xie, L. Sun, Q. Fatima, Int. J. Hydrog. Energy 43 (2018) 21114.

[7] M. Thepnurat, T. Chairuangsri, N. Hongsith, P. Ruankham and S. Choopun, ACS Appl. Mater. Interfaces 7 (2015) 24177.

[8] C. Wang, Z.-G. Wang, R. Xi, L. Zhang, S.-H. Zhang, L.-J. Wang and G.-B. Pan, Sens. Actuators B Chem. 292 (2019) 270.

[9] S. Park, T. Hong, J. Jung and C. Lee, Curr. Appl. Phys. 14 (2014) 1171.

[10] G. Lu, J. Xu, J. Sun, Y. Yu, Y. Zhang and F. Liu, Sens. Actuators B Chem. 162 (2012) 82.

[11] M. V. Vaishampayan, R. G. Deshmukh, P. Walke and I. S. Mulla, Mater. Chem. Phys. 109 (2008) 230.

[12] H.M. Tan, C. Manh Hung, T.M. Ngoc, H. Nguyen, N. Duc Hoa, N. Van Duy and N.V. Hieu, ACS. Appl. Mater. Interfaces 9 (2017) 6153.

[13] R. Dhahri, M. Hjiri, L.E. Mir, A. Bonavita, D. Iannazzo, S.G. Leonardi and G. Neri, Appl. Surf. Sci. 355 (2015) 1321.

[14] D. Haridas and V. Gupta, Sens. Actuators B Chem. 182 (2013) 741.

[15] H. Chen, Y. Liu, C. Xie, J. Wu, D. Zeng and Y. Liao, Ceramics International. 38 (2012) 503.

[16] A. Giberti, C. Malagù and V. Guidi, Sens. Actuators B Chem. 165 (2012) 59.

[17] J. D. Prades, R. Jimenez-Diaz, F. Hernandez-Ramirez, S. Barth, A. Cirera, A. Romano-Rodriguez, S. Mathur and J. R. Morante, Sens. Actuators B Chem. 140 (2009) 337. 
[18] P. Fageria, S. Gangopadhyay and S. Pande, RSC Advances 4 (2014) 24962.

[19] W. Muhammad, N. Ullah, M. Haroon and B. H. Abbasi, RSC Adv. 9 (2019) 29541.

[20] A.G. Saputro, F.T. Akbar, N.P.P. Setyagar, M.K. Agusta, A. D. Pramudya and H.K. Dipojono, New J. Chem. 44 (2020) 7376.

[21] M.J.S. Spencer and I. Yarovsky, J. Phys. Chem. C 114 (2010) 10881.

[22] V. Srikant and D.R. Clarke, J. Appl. Phys. 83 (1998) 5447.

[23] K. Davis, R. Yarbrough, M. Froeschle, J. White and H. Rathnayake, RSC Adv. 9 (2019) 14638.

[24] T. Weis, R. Lipperheide, U. Wille, S. Brehme, J. Appl. Phys. 92 (2002) 1411. 